\title{
Improving Usability of Search and Rescue Decision Support Systems: WARA-PS Case Study
}

Veronika Domova, Erik Gärtner, Fredrik Präntare, Martin Pallin, J ohan Källström and Nikita Korzhitskii

The self-archived postprint version of this journal article is available at Linköping University Institutional Repository (DiVA):

http:// urn.kb.se/ resolve?urn=urn:nbn:se:liu:diva-170328

N.B.: When citing this work, cite the original publication.

Domova, V., Gärtner, E., Präntare, F., Pallin, M., Källström, J., Korzhitskii, N., (2020), Improving Usability of Search and Rescue Decision Support Systems: WARA-PS Case Study, In proceedings of the 2020 25th IEEE International Conference on Emerging Technologies and Factory Automation (ETFA), , 1251-1254. https:// doi.org/ 10.1109/ETFA46521.2020.9211980

Original publication available at:

https:/ doi.org/ 10.1109/ETFA46521.2020.9211980

Copyright: IEEE conference proceedings

http:// www.ieee.org//

(C)2020 IEEE. Personal use of this material is permitted. However, permission to reprint/republish this material for advertising or promotional purposes or for creating new collective works for resale or redistribution to servers or lists, or to reuse any copyrighted component of this work in other works must be obtained from the IEEE. 


\title{
Improving Usability of Search and Rescue Decision Support Systems: WARA-PS Case Study
}

\author{
Veronika Domova $^{1}$, Erik Gärtner ${ }^{2}$, Fredrik Präntare ${ }^{1}$, Martin Pallin ${ }^{3}$, Johan Källström ${ }^{1}$, Nikita Korzhitskii ${ }^{1}$ \\ ${ }^{1}$ Linköping University, Linköping, Sweden, \{verdo29, fredrik.prantare, johan.kallstrom, nikita.korzhitskii\}@liu.se \\ ${ }^{2}$ Lund University, Lund, Sweden, erik.gartner@math.lth.se \\ ${ }^{3}$ Royal Institute of Technology, Stockholm, Sweden, pallin@kth.se
}

\begin{abstract}
Novel autonomous search and rescue systems, although powerful, still require a human decision-maker involvement. In this project, we focus on the human aspect of one such novel autonomous SAR system. Relying on the knowledge gained in a field study, as well as through the literature, we introduced several extensions to the system that allowed us to achieve a more user-centered interface. In the evaluation session with a rescue service specialist, we received positive feedback and defined potential directions for future work.

Index Terms-Public Safety, Search and Rescue, Control System, User Interface
\end{abstract}

\section{INTRODUCTION}

Public Safety (PS), i.e. safeguarding the population from disasters, often means dealing with extreme situations that are dangerous not only for the general public but also for the rescue team. Therefore, reducing the number of participating in-person humans through the involvement of autonomous search and rescue (SAR) systems is of great interest [1], [2]. However, the adoption of autonomous solutions into SAR scenarios does not eliminate the necessity of human involvement. The role of the human is changing from being an active participant in rescue missions to a remote operator making high-order decisions remotely. The operator's situation awareness (SA) is a crucial notion on which these decisions and overall mission safety and performance hinge [3]. A major portion of the operator's job becomes that of obtaining and maintaining good SA, which often becomes a struggle [4]. Supporting SAR operators in maintaining high SA becomes essential.

In this paper, we present our work on improving SA of the operator of a novel SAR control system, i.e. WARA-PS demonstrator. Our project started with field studies at a Fire and Rescue Services center to gain knowledge about SAR operators' tools, workflows, problems, and needs. Based on the obtained knowledge, we identified requirements to the control system in focus and extended it with the following features: a) an AI-powered image recognition to classify objects in the field media data, b) a 3D visualization of the WARA-PS archipelago area to provide an immersive experience, and c) an enhanced look-and-feel of the user interface to improve SA.

This work was supported by the Wallenberg AI, Autonomous Systems and Software Program (WASP) funded by the Knut and Alice Wallenberg Foundation. We would like to thank Jesper Tordenlid for all the support in the project.
In this paper, we elaborate on the formulated requirements, describe the delivered extensions, report on the preliminary evaluation results, and discuss future research potential.

\section{FIELD STUDY}

In the scope of the field studies, we have visited a Fire and Rescue Services center located in a small-sized Swedish coastal town. The organization mainly coordinates missions related to fire, traffic and CBRNE (Chemical, Biological, Radiological, Nuclear and Explosive) accidents at land and on water.

The missions of the organization are managed by operators in a local command and control center. A working place of an operator comprises a desk with multiple screens hosting the control system. The interface of the control system comprises a) a 2D map showing ongoing missions and related geographical information, b) a tabbed interface showing additional textual information about the ongoing missions. In addition, a variety of other map-based tools, simulators and sources of information are used on a daily basis. Most missions are of small-scale and are rather typical and easy to deal with, e.g. traffic accidents, fires in small buildings, and small fires in nature. The most commonplace large-scale missions are related to handling forest fires and leakages of environmentally dangerous substances at sea. They are more complicated because it is challenging to 1) get a good overview of the state of such a mission due to its scale, 2) ensure that all personnel has the same SA. Incomplete information, which is a common case, makes it difficult to locate the accident accurately and act appropriately.

SAR operations are subject to a high degree of uncertainty due to various changing factors that can make the task at hand more or less difficult. One important aspect that can heavily influence the flow and outcome of a mission is the weather. A similar influence has the geographical location of the mission and the type of terrain at the location because some areas simply cannot be reached by certain types of vehicles. A significant factor is the distance from the accident location to the closest rescue team defining how fast the team can arrive at the accident location. Finally, concurrently running missions can add a great deal of complexity because one needs to maintain a good SA about each accident and make decisions about assigning limited resources to one or another mission. 


\section{WARA-PS PROJECT}

In Sweden, several industrial companies and universities have joined forces in a common effort to develop a research arena, i.e. Wallenberg Research Arena - Public Safety (WARA-PS $)^{1}$, for testing autonomous SAR scenarios at sea. The idea is to use autonomous agents, such as unmanned surface vehicles (USVs), unmanned aerial vehicles (UAVs), as well as human agents, control systems and other advanced technical solutions in order to autonomously search, locate and rescue distressed persons in danger. A suitable location in the Swedish archipelago was selected, where the physical setup for testing SAR scenarios is being arranged.

WARA-PS control system, which is in the focus of the current paper, is intended to a) autonomously coordinate field agents, i.e. plan and execute the flow of search and rescue missions, b) provide an interface for control room operators, so that they can monitor ongoing missions and, if necessary, introduce manual corrections into the flow of ongoing missions. At the moment, the system does not have a real communication channel with the fleet of field agents. Instead, a simulator replaces all the field assets. The simulator plans and simulates missions; information about the missions and their flow is depicted on the UI.

When a simulation of a mission starts, involved agents are directed to the accident area to perform the search according to their plan. The search logic is arranged as follows: 1) the accident area is divided into cells, 2) agents are assigned to scan different cells according to their plans, 3) as soon as an agent reaches and scans a cell, the latter is considered as searched. The data collected by the agents, e.g. images, is preprocessed by the system and visualized on the UI in the form of notifications, that can then be manually inspected by the user. The UI, see Figure 1, consists of a 2D map and a sliding tabbed panel, which provides access to more information and actions on demand. The map depicts the archipelago view, ongoing mission(s) if any, as well as the locations of field agents. The sliding panel provides information about a) ongoing missions, $b$ ) the available fleet, c) notifications from the ongoing missions, e.g. the images captured by the field agents, and d) weather information. The panel also allows creating missions and initiating their simulations.

Our analysis revealed several omissions of the UI that can potentially lead to poor user experience and performance in the future. First of all, the existing interface does not allow the user to get an at-a-glance overview of the ongoing missions or of the available/busy fleet without clicking through the tabs. The map, occupying the central place of the interface, is of too little help either as it is challenging to compare the progress of different missions or prioritize one mission over another using the means of a map. Moreover, the system in its current state assumes manual processing of incoming field data, which would inevitably put an extra load on the operator. This paper describes efforts undertaken in an attempt

\footnotetext{
${ }^{1}$ https://wasp-sweden.org/demonstrators/wara-ps-public-safety
}

to address the aforementioned issues and make the interface more user-oriented.

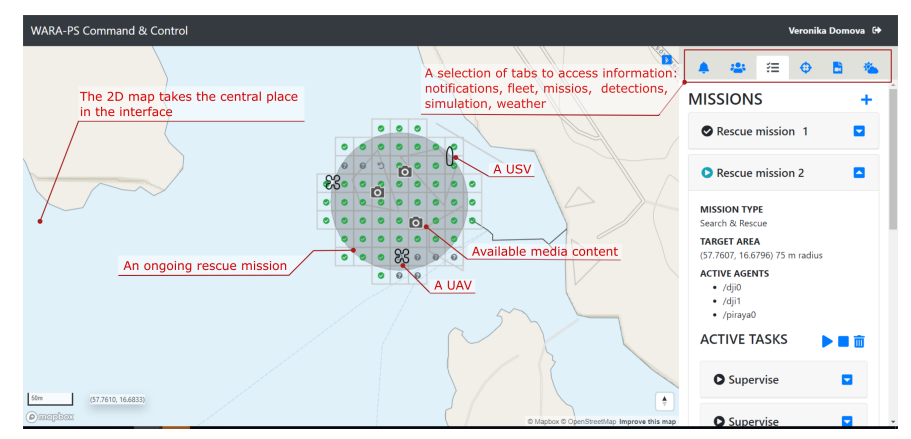

Fig. 1. The original interface of the system. The map depicts one ongoing mission. The mission area is marked with a light-gray circle. Sectors are shown as squares. Visited sectors are marked with green ticks, not visited with a question mark, sectors that should be revisited are marked with a round arrow. The locations of field agents, as well as the availability of media data, are shown by corresponding icons.

\section{RELATED WORK}

Several works come up with interface design implications for improved SA in SAR scenarios, namely a) the interface should be capable of providing a complete and quick to interpret overview of the rescue mission [5], b) maps should be rather simplified with no extra markers or pointers to support better readability [6], c) immersive experiences based on Virtual Reality (VR) technologies have a positive effect on operators' SA [7]. The majority of SAR interfaces reported in relevant works [3], [5], [8], [9] use some sort of a dashboard to present information and enable control. Interfaces of earlier SAR systems, i.e. with lower levels of automation requiring manual control of field agents, [3], [5] were focusing mostly on one agent at a time; their interfaces included three major information areas, i.e. the live feed from the agent's camera (if available), a 2D or 3D map with positioning information, and status information of the agent with its control interface. Dashboards of more recent SAR systems [8], [9] with semior fully autonomous agents tend to focus on a larger picture of an ongoing mission.

With respect to the works discovered, the novelty of our work is that a) we target SAR systems with a fully autonomous fleet, which normally would not require manual assistance in basic tasks such as navigation and path planning, b) we aim to support SAR operators that manage large fleets and monitor multiple missions ongoing simultaneously.

\section{WARA-PS CONTROL SYSTEM INTERFACE REQUIREMENTS}

Based on the knowledge gained from the field studies, as well as from the literature review, we identified a set of interface requirements specific to the WARA-PS control system; each of them is marked with $\mathbf{R}$ letter and a number for the purpose of further referencing in this paper.

First of all, (R1) the interface should provide an at-a-glance overview of the ongoing missions and the fleet, as well as other 
relevant information, such as the weather, geospatial data, etc. Importantly, the interface should be flexible and capable to visualize information about many ongoing missions spread on a large geographical area and depict large fleets consisting of hundreds or even thousands of agents. Second, (R2) the interface should allow comparison and prioritizing, because prioritization is one of the critical tasks in SAR operators' work [10]. In conditions of limited resources, i.e. available field agents, and multiple missions ongoing in parallel, the operators will have to decide, which mission should get priority. Third, (R3) there should be a possibility to drill down into details, e.g. get sufficient SA about the progress of a particular mission; the user should be well informed to be able to manually change the autogenerated mission plan. Finally, taking into account missions ongoing in parallel and a multitude of field agents, it will be unfeasible for the operator to manually process all the data being received. Therefore, (R4) it is essential to apply data aggregation and pre-processing means before the data is getting rendered on the UI.

\section{SySTEM EXTENSIONS}

\section{A. Dashboard}

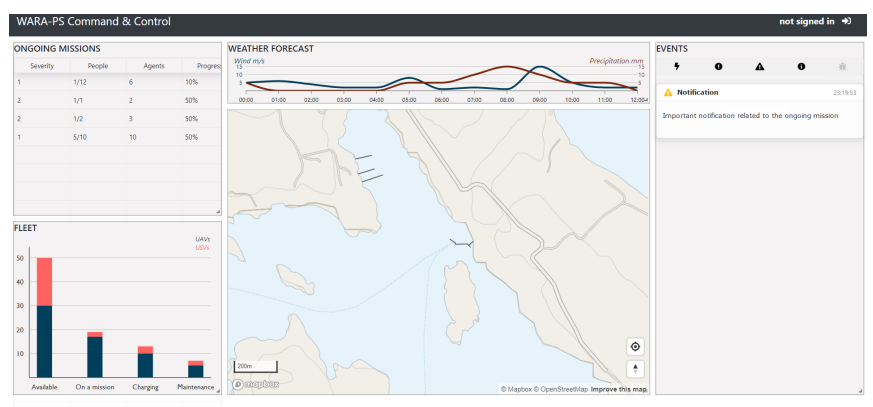

Fig. 2. The dashboard.

We have designed and developed an interactive dashboard which is intended to serve as the starting point for the operator's workflows, see Figure 2. The dashboard aggregates and presents all the data that was initially available in the original interface, see Figure 1, but it allows to observe it all at once (R1). The dashboard layout is flexible, i.e. the tiles can be resized, moved around, deleted or added. At the moment, the dashboard consists of 5 tiles, namely: 1) Ongoing missions tile contains a list of ongoing missions and their most important attributes in a tabulated format which simplifies comparison between the missions (R2); 2) Fleet tile contains a bar chart summarizing the current status of the fleet, i.e. how many agents are available at the moment, how many are occupied, how many are on charge, and how many are undergoing maintenance; the graphical representation allows to reduce the amount of visual information on the screen (R4), and to depict the status of a fleet of any size (R1); 3) Weather forecast tile contains a line graph showing the weather forecast, i.e. wind and precipitation, for the next 12 hours; 4) Map tile contains the original 2D map of the WARAPS arena archipelago; 5) Events tile contains the list of events and notifications.

\section{B. AI-powered Object Detection}

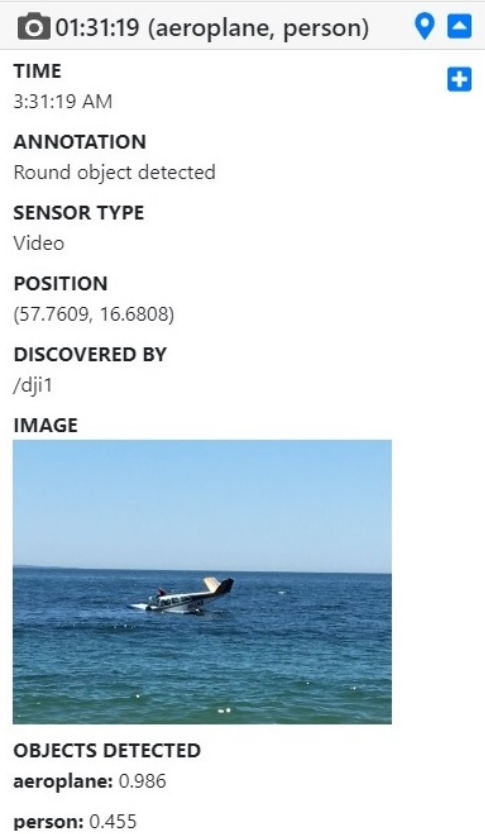

Fig. 3. An example of AI-powered object detection.

We have introduced AI assistance for filtering and prioritizing incoming information in an attempt to reduce the flow of information and facilitate the operator's decision making (R4). For this purpose, we integrated into the system a commonly used object detector YOLOv3 [11]. Next, we collected a small proof-of-concept dataset of images resembling a potential output from the field agents surveying an incident area in a sea, e.g. photos of boat incidents, drowning or swimming people, views of open water and the archipelago, etc. When simulating a mission, $\mathrm{C} 2$ simulator randomly assigns images from the dataset to visited sectors of the incident area as if they were taken by the field agents. The object detector analyzes and annotates incoming images with information regarding the content and confidence. This information is then depicted on the UI, see Figure 3. The user is getting explicitly notified by visual cues only about images containing detections of interest. As a result, the amount of data directly requiring the attention of the operator is reduced $(\mathbf{R 4})$.

\section{C. $3 D$ Interface}

We developed a 3D visualization of the WARA-PS archipelago to enable 3D visualizations of SAR missions. We took an open-source 3D model of the archipelago area where the WARA-PS arena is located as the basis of the 3D visualization and elaborated it by adding the water surface. The $3 \mathrm{D}$ visualization is integrated with the baseline control system, it is subscribed to updates of the positions of UAVs 
and USVs, as well as information about detections made by field agents. The information is then displayed as 3D objects in the 3D scene, see Figure 4. The 3D visualization is available on-demand when the user wants to get better SA about a particular ongoing mission (R3).

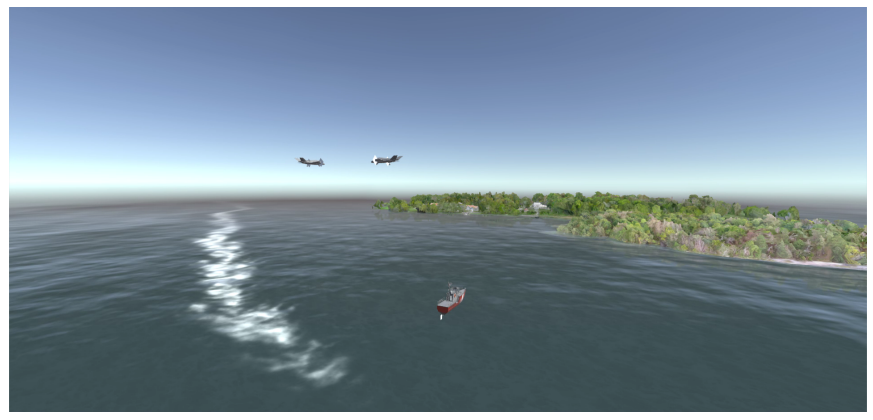

Fig. 4. A screenshot of the 3D visualization: two UAVs and one USV are in the process of scanning the area.

\section{Preliminary EVAluation}

A preliminary evaluation session was conducted with a specialist in fire prevention from the original Fire and Rescue Services center. First, the developed extensions were demonstrated and explained to the user. Then their preliminary feedback, comments, and proposals were noted down.

In general, the interviewee was very positive about all the presented innovations and could see potential application cases in their daily routines. With respect to the AI-powered image recognition and information extraction, he agreed that such solutions would free up operators and allow them to focus on other tasks rather than manually go through field data. The interviewee concluded that he would trust an AI system as long as he could review all the information himself and as long as there is a human in the loop that makes the final decision.

With regard to the interactive 3D model of the archipelago, the interviewee saw a special potential for using it for exploring terrain specifics of accident locations or for inspecting the details of accident scenes. For example, approaching a burning tanker is dangerous, whereas estimating the damage from 2D images can be hard. Therefore, having a possibility to quickly scan the environment with drones and create a 3D reconstruction of the site would let the firemen inspect the incident on a tablet or in VR, reveal the source(s) of the fire, and proceed accordingly.

Assessing the dashboard, the interviewee confirmed that it would be beneficial to have a modular interface, similar to the presented dashboard, where only relevant information to a particular accident would be shown. A more advanced option would be to make the interface automatically adapt to the accident type and configure the UI elements in correspondence with real-time information coming from the field. For example, now, in the case of chemical leakage, to obtain a sufficient SA, the operator first needs to bring up the toxin dispersion map and then manually calibrate a set of parameters. An interface that could do it automatically would come in handy.

\section{CONCLUSION AND FUtURE WORK}

Initiatives similar to WARA-PS foretell that the adoption of higher levels of automation will introduce significant changes in SAR operator's workflows, namely a) there will be less manual control and more strategic actions, b) there will be more data coming from the field to handle. As such, more effort is needed to provide operators with a new generation of control tools. The project described in this paper is an applied example of such an effort. In its scope, we relied on the gained domain knowledge, as well as on the conducted literature study, to formulate a set of design requirements for SAR control systems of high autonomy. The main result of our work is a set of extensions, that improved the original interface of the WARA-PS control system and made it more user-oriented. The extensions aim to help the operator obtain the necessary information to maintain sufficient SA. The preliminary evaluation with a real user showed that the developed extensions were received positively and led to multiple recommendations and ideas for future work. The next steps will incorporate connecting the WARA-PS control system to the actual fleet of field agents and filling the interface with real data from the field. The final step is to run a long-term evaluation of the system in real settings.

\section{REFERENCES}

[1] G. De Cubber, D. Doroftei, D. Serrano, K. Chintamani, R. Sabino, and S. Ourevitch, "The eu-icarus project: developing assistive robotic tools for search and rescue operations," in 2013 IEEE international symposium on safety, security, and rescue robotics (SSRR). IEEE, 2013, pp. 1-4.

[2] S. Yeong, L. King, and S. Dol, "A review on marine search and rescue operations using unmanned aerial vehicles," Int. J. Mech. Aerosp. Ind. Mech. Manuf. Eng, vol. 9, no. 2, pp. 396-399, 2015.

[3] J. M. Riley and M. R. Endsley, "The hunt for situation awareness: Human-robot interaction in search and rescue," in Proceedings of the Human Factors and Ergonomics Society Annual Meeting, vol. 48, no. 3. SAGE Publications Sage CA: Los Angeles, CA, 2004, pp. 693-697.

[4] J. L. Burke, R. R. Murphy, M. D. Coovert, and D. L. Riddle, "Moonlight in miami: Field study of human-robot interaction in the context of an urban search and rescue disaster response training exercise," HumanComputer Interaction, vol. 19, no. 1-2, pp. 85-116, 2004.

[5] F. Driewer, H. Baier, and K. Schilling, "Robot-human rescue teams: a user requirements analysis," Advanced Robotics, vol. 19, no. 8, pp 819-838, 2005.

[6] P. L. McDermott, R. Chadwick, J. M. Riley, and A. Fisher, "Providing the right amount of detail: Implications for map and icon display in a robotic search task," in Proceedings of the Human Factors and Ergonomics Society Annual Meeting, vol. 55, no. 1. SAGE Publications Sage CA: Los Angeles, CA, 2011, pp. 449-453.

[7] J. J. Roldán, E. Peña-Tapia, D. Garzón-Ramos, J. de León, M. Garzón, J. del Cerro, and A. Barrientos, "Multi-robot systems, virtual reality and ros: Developing a new generation of operator interfaces," in Robot Operating System (ROS). Springer, 2019, pp. 29-64.

[8] A. Agrawal, S. Abraham, B. Burger, C. Christine, L. Fraser, J. Hoeksema, S. Hwang, E. Travnik, S. Kumar, W. Scheirer et al., "The next generation of human-drone partnerships: Co-designing an emergency response system," arXiv preprint arXiv:2001.03849, 2020.

[9] A. Hong, O. Igharoro, Y. Liu, F. Niroui, G. Nejat, and B. Benhabib, "Investigating human-robot teams for learning-based semi-autonomous control in urban search and rescue environments," Journal of Intelligent \& Robotic Systems, vol. 94, no. 3-4, pp. 669-686, 2019.

[10] H. R. Ranjbar, A. A. Ardalan, H. Dehghani, and M. R. Saradjian, "A proposed spatial index to prioritize damaged buildings for allocating usar operations," Geocarto international, vol. 33, no. 8, pp. 791-809, 2018.

[11] J. Redmon and A. Farhadi, "Yolov3: An incremental improvement," CoRR, vol. abs/1804.02767, 2018. [Online]. Available: http://arxiv.org/abs/1804.02767 